\title{
Apelin-APJ Signaling: a Potential Therapeutic Target for Pulmonary Arterial Hypertension
}

\author{
Jongmin Kim*
}

Pulmonary arterial hypertension (PAH) is a progressive disease characterized by the vascular remodeling of the pulmonary arterioles, including formation of plexiform and concentric lesions comprised of proliferative vascular cells. Clinically, PAH leads to increased pulmonary arterial pressure and subsequent right ventricular failure. Existing therapies have improved the outcome but mortality still remains exceedingly high. There is emerging evidence that the seven-transmembrane G-protein coupled receptor APJ and its cognate endogenous ligand apelin are important in the maintenance of pulmonary vascular homeostasis through the targeting of critical mediators, such as Krúppel-like factor 2 (KLF2), endothelial nitric oxide synthase (eNOS), and microRNAs (miRNAs). Disruption of this pathway plays a major part in the pathogenesis of PAH. Given its role in the maintenance of pulmonary vascular homeostasis, the apelin-APJ pathway is a potential target for PAH therapy. This review highlights the current state in the understanding of the apelin-APJ axis related to PAH and discusses the therapeutic potential of this signaling pathway as a novel paradigm of PAH therapy.

\section{INTRODUCTION}

Pulmonary arterial hypertension (PAH) is a devastating disease localized to the small pulmonary arterioles, which is characterized by increased pulmonary vascular resistance and right ventricular failure. The latter is the most common cause of death. PAH is clinically defined by an elevation in mean pulmonary artery pressure exceeding $25 \mathrm{mmHg}$ at rest (Simonneau et al., 2009). PAH is histologically characterized by endothelial and smooth muscle cell proliferation, medial hypertrophy, muscularization of the arterioles, and thrombosis in situ (Rabinovitch et al., 2007). The etiology of PAH is complex and largely undetermined, and it remains a fatal disease if left untreated.

Department of Life Systems Sookmyung Women's University, Seoul 140-742, Korea

${ }^{*}$ Correspondence: jkim@sookmyung.ac.kr

Received 24 October, 2013; revised 28 November, 2013; accepted 2 December, 2013; published online 3 March, 2014

Keywords: apelin-APJ pathway, endothelial cell microRNA, proliferation, pulmonary arterial hypertension
Currently, there are few approved drugs for the treatment of $\mathrm{PAH}$. Most such as endothelin-1 (ET-1) receptor antagonists, prostacyclin analogues, and phosphodiesterase- 5 inhibitors, are primarily pulmonary vasodilators that maintain the balance of endothelium-derived vasoactive mediators (McLaughlin and McGoon, 2006). However, despite these advances, PAH remains a fatal disease without curative therapies. Mortality rates remain exceedingly high with $15 \%, 30 \%$, and $45 \%$ mortality at 1,2 , and 3 years after diagnosis, respectively (Humbert et al., 2010). Identifying novel targets for PAH therapy is crucial.

The aim of most current therapies is to improve hemodynamic parameters and functional lung capacity through vasodilatation of the pulmonary vasculature. Emerging data suggests that targeting abnormal proliferation of vascular cells may, in fact, be a viable and efficacious therapeutic option. In line with this possibility, disruption of apelin-APJ signaling was recently proven to be involved in vascular remodeling via abnormal proliferation of vascular cells, as well as in the progression of $\mathrm{PAH}$ (Kim et al., 2013).

This review discusses the links between apelin-APJ signaling and pathogenesis of $\mathrm{PAH}$, and its possibility as a potential therapeutic target for $\mathrm{PAH}$.

\section{APELIN-APJ SIGNALING IN VASCULAR BIOLOGY}

\section{Characterization of apelin and APJ}

The APJ receptor was first cloned from a human gene based on homology to other known $G$ protein coupled receptors (O'Dowd et al., 1993). The human APJ (approved gene symbol: $A P L N R$ ) gene encodes a 377 amino acid G-protein coupled receptor with seven transmembrane domains and shares $31 \%$ sequence identity at the protein level with the Angiotensin II receptor type I (AT1R). However, the APJ receptor does not bind with angiotensin II (Ang II).

The ligand for APJ was identified in 1998 and was named apelin (Tatemoto et al., 1998). Initially characterized as a 36 amino acid peptide, cloning of the cDNA identified it as a prepro-protein of 77 amino acids that is cleaved to generate shorter biological active peptides of 13 and 17 amino acids that exist in vivo (Hosoya et al., 2000; Kawamata et al., 2001; Tatemoto et al., 1998). These shortened forms have more potent effects than the longer form (Tatemoto et al., 1998). A pyroglutamylated form of apelin-13 is produced endogenously improves resistance to enzymatic cleavage, and has been more recently used experimentally as a more suitable peptide for chronic infusion. 
Tissue and cellular distribution of apelin and APJ

Multiple tissues express apelin and APJ, including the heart, lungs, and vasculature (Medhurst et al., 2003). In the cardiovascular system, animal studies using mice and rat tissues have documented the elevated expression of both apelin and APJ messenger RNA (mRNA) in the heart and lungs (Medhurst et al., 2003), and apelin has been localized to endothelial cells associated with small vessels in various tissues (Sheikh et al., 2008; Tatemoto et al., 2001).

\section{Apelin-APJ signaling in cardiovascular physiology}

The apelin-APJ pathway appears to modulate cardiac contractility and vascular tone. Apelin is an arterial and venous dilator in vivo (Cheng et al., 2003; Lee et al., 2000; Tatemoto et al., 1998), and induces a potent dose-dependent positive inotropic effect in isolated perfused rat hearts (Szokodi et al., 2002). In vivo, apelin infusions for two weeks improves cardiac contractility and reduces cardiac loading (Ashley et al., 2005). More recent studies have demonstrated a link between apelin and Ang II signaling pathways. The apelin-APJ pathway mediates opposing actions to the renin-angiotensin system (RAS) in a number of physiologic and pathophysiologic settings. Indeed, while Ang II increases vascular tone and raises blood pressure, apelin is a vasodilator and lowers blood pressure; in heart failure, Ang II levels rise, whereas apelin levels fall (Chen et al., 2003; Cheng et al., 2003; De Mota et al., 2004; Ishida et al., 2004; Lee et al., 2000; O'Carroll et al., 2006; Tatemoto et al., 1998). The apelin-APJ pathway also appears to physiologically antagonize RAS, suggesting that apelin-APJ signaling can suppress Ang II actions in vascular disease (Chun et al., 2011). In addition, there is evidence for direct counter-regulation of apelin-APJ signaling by RAS. Differences in blood pressure response in mice lacking both the AT1R and APJ, compared to mice lacking only AT1R were described (Ishida et al., 2004) and Ang II inhibits expression of apelin and APJ, perhaps by contributing to cardiac decompensation in a hypertensive model of heart failure (Iwanaga et al., 2006). Most recently, Kang et al. (2013) demonstrated that apelin-APJ signaling plays an important role in cardiovascular development, controlling the activation of myocyte enhancer factor-2 (MEF2) transcription factors by regulating $\mathrm{G}$ alpha 13 , histone deacetylase (HDAC) 4 , and HDAC5.

\section{APELIN-APJ SIGNALING IN PAH}

Vascular remodeling in $\mathrm{PAH}$

The pathogenesis of $\mathrm{PAH}$ is a multifactorial process leading to functional and structural changes in the pulmonary vasculature. Common histopathologic patterns include: 1) intimal hyperplasia, 2) medial hypertrophy, 3) adventitial proliferation/fibrosis, 4) occlusion of small arteries, 5) thrombosis in situ, and 6) plexiform lesions comprised of hyperproliferative pulmonary artery endothelial cells (PAECs) and pulmonary artery smooth-muscle cells (PASMCs) (Rai et al., 2008; Sakao et al., 2010). The majority of cases are sporadic, but genetic studies in cases of familial PAH have identified the bone morphogenetic protein receptor type II (BMPR2) gene as the gene for familial PAH (Machado et al., 2009).

Although the exact causes of PAH remain for the most part unknown, endothelial dysfunction is likely an important contributor to the progression of the disease (Budhiraja et al., 2004). The endothelial disruption that occurs in $\mathrm{PAH}$ is complex, and is thought to manifest in part by the imbalance of locally produced vasoconstrictors and vasodilators, including nitric oxide
(NO), prostacyclin, endothelin-1 (ET-1), serotonin, and thromboxane. Clinical studies have demonstrated perturbation in these factors in the lungs of patients with PAH (Ozkan et al., 2001; Raja et al., 2010). The vascular remodeling results from dysregulation of endothelial and vascular smooth muscle cell growth. Abnormal proliferation of endothelial cells is seen in the plexogenic lesions expressing angiogenic factors, such as vascular endothelial growth factor (VEGF) and basic fibroblast growth factor 2 (FGF2) (Benisty et al., 2004; Izikki et al., 2009). These factors from endothelial cells affect smooth muscle cells growth in a paracrine manner (Kim et al., 2013). In addition, the pulmonary vascular smooth muscle cells also undergo proliferation and hypertrophy. These changes seem to be mediated by loss of anti-mitogenic factors and increase in mitogenic factors. The significant evidence sup-porting the importance of endothelial disruption in PAH makes this cell type an ideal target for therapeutic development in $\mathrm{PAH}$ patients. This novel paradigm involving a link between dysfunctional endothelial cells and apelin-APJ axis in these cells may have important implications for understanding the pathogenesis of $\mathrm{PAH}$ and possibly lead to more effective treatments (Fig. 1).

\section{$\mathrm{PAH}$ and cancer paradigm}

$\mathrm{PAH}$ involves abnormal proliferation of multiple cell types, such as PAECs and PASMCs, in the pulmonary vasculature. Dysregulated cellular growth leads to formation of vascular plexiform lesions, which is the hallmark of severe PAH. These plexiform lesions are composed of hyperproliferative PAECs and PASMCs. The concept of the endothelial cell as "quasi-malignant" was initially suggested in 1998 (Rai et al., 2008; Tuder et al., 2001) and has been the focus of more recent attention in the context of clinical trials demonstrating efficacy of anti-cancer therapies, such as tyrosine kinase inhibitors in the treatment of $\mathrm{PAH}$ (Barst, 2005; Ghofrani et al., 2010). The complex vascular lesions of PAH appear to be governed by the same traits that control cancer growth, including angiogenesis, absence of apoptotic cells in the lesions, and the presence of antiapoptotic proteins in the lesion cells (Hanahan and Weinberg, 2000). These studies have provided support for targeting aberrant cellular proliferation in treatment of $\mathrm{PAH}$.

Given the similarities between hyperproliferative phenotype in cancer and $\mathrm{PAH}$, the most recent study is germane. The study showed that miR-424 and miR-503 regulated by apelinAPJ signaling in PAECs exert anti-proliferative effects and inhibit the proliferation of PASMC in a paracrine manner, which may bridge the current gap in PAH specific mechanism concerning how these PAECs undergo a switch from a quiescent homeostatic state to a hyperproliferative state and affect PASMC growth (Kim et al., 2013). These data provide support for targeting aberrant cellular proliferation in treatment of PAH (Figs. 1 and 2).

\section{Regulation of apelin signaling by BMPR2 and hypoxia} BMPR2 is a member of the transforming growth factor beta (TGF- $\beta$ ) superfamily of growth factor receptors. Mutations in this gene are causally linked to $\mathrm{PAH}$, where loss-of-function mutations in the BMPR2 gene have been found in approximately $70 \%$ of $\mathrm{PAH}$ patients with a family history of $\mathrm{PAH}$, in 10$40 \%$ of PAH patients without a family history of the disease (Deng et al., 2000; Machado et al., 2009; Simonneau et al., 2009), and in $6-9 \%$ of patients with secondary forms of $\mathrm{PAH}$ associated with a number of other conditions, including connective tissue disease and congenital heart disease. However, the penetrance of heritable PAH is low; on average, only $15 \%$ of 


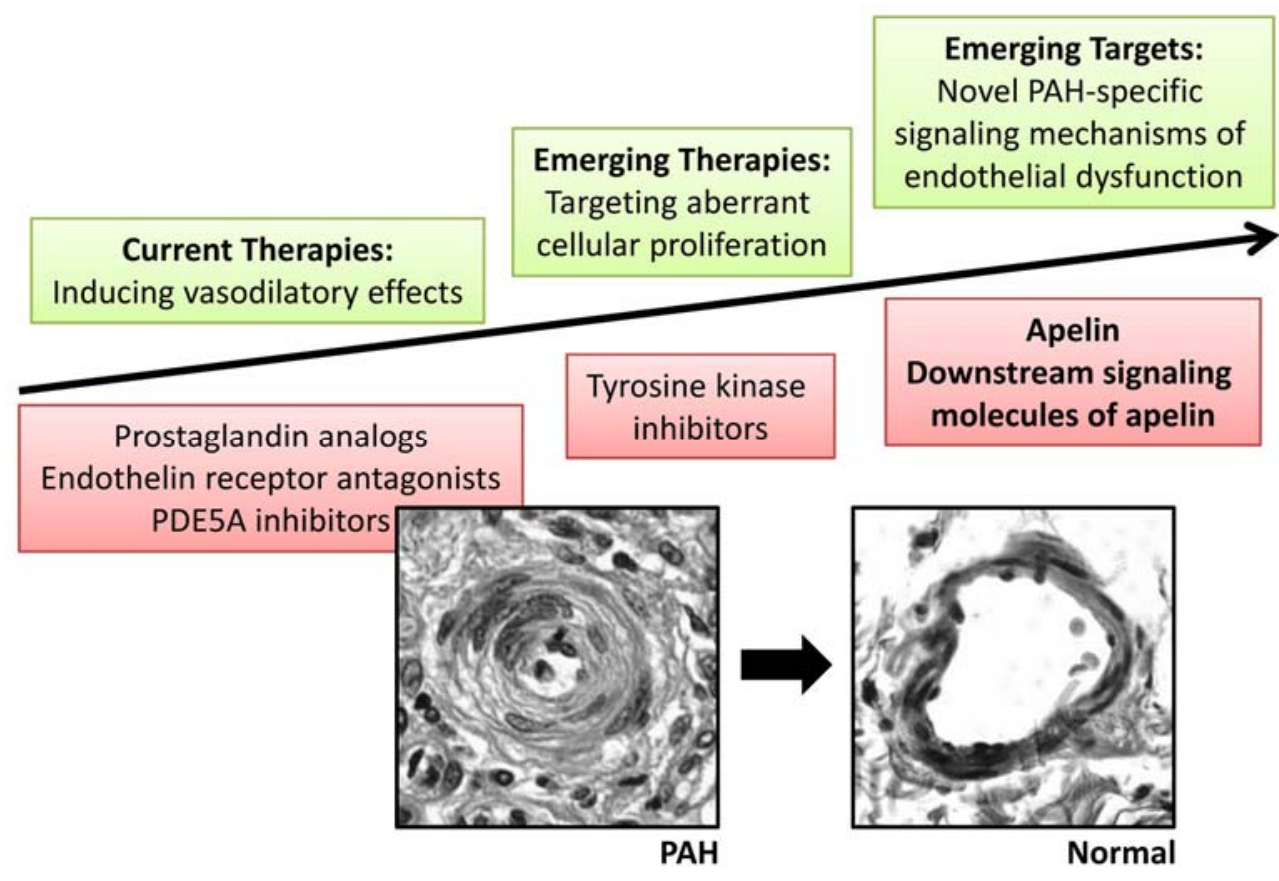

Fig. 1. Proposed novel paradigms for $\mathrm{PAH}$ therapy. Augmentation of apelinAPJ signaling in endothelial cells might be an attractive therapeutic venue for $\mathrm{PAH}$ treatment. affected family members develop PAH (Hamid et al., 2009; Machado et al., 2009; Roberts et al., 2004), suggesting the importance of abnormalities in downstream signaling of BMPR2 or environmental influences that could disrupt BMPR2 function through the inhibition of its expression, thereby contributing to PAH development (Hansmann et al., 2007; Zamanian et al., 2009). Recently, apelin signaling was identified as a key downstream target in pulmonary vascular homeostasis of the BMPR2 signaling (Alastalo et al., 2011). Given that dysfunctional BMPR2 signaling is involved in the pathogenesis of $\mathrm{PAH}$, the authors showed that BMPR2 signaling mediated by a complex between $\beta$-catenin and peroxisome proliferator activated receptor gamma (PPAR $\gamma$ ) is able to regulate apelin expression. The authors also found that disruption of a complex between $\beta$-catenin and PPAR $\gamma$ through abnormal BMPR2 signaling leads to decreased apelin expression. In turn autocrine effects result in increased PAECs apoptosis, as well as paracrine effects in suppression of abnormal proliferation of PASMCs. The authors further reported reversal of PAH by exogenous apelin peptide administration in PPAR $\gamma$ knockout mice, suggesting that apelin could be a beneficial target in treating $\mathrm{PAH}$ through the restoration of BMPR2 signaling.

Hypoxia-induced pulmonary vasoconstriction is a well-established animal model of pulmonary arterial hypertension (Zaidi et al., 2002). Apelin expression is significantly increased by hypoxia in cultured cell lines, including human coronary artery endothelial cells, human microvascular endothelial cells, and human PAECs (Sheikh et al., 2008). A recent study also demonstrated that mRNA expression of heart and lung resulted in the marked upregulation of apelin and APJ in mice after 1 week of hypoxia. However, after 3 weeks of hypoxia, there was a significant reduction in the expression of both genes (Chandra et al., 2011). These findings suggest that the initial up-regulation of apelin and APJ during hypoxia may reflect a compensatory mechanism that antagonizes the vasoconstrictive mediators of hypoxia. However, with prolonged hypoxia, this pathway is down-regulated, leading to a failure to compensate for the progressive vasoconstriction and pulmonary vasculature remodeling

Disruption of apelin-APJ signaling in PAH

Recent studies have focused on the endothelial signaling pathways that are disrupted in PAH. Disruption of apelin-APJ signaling pathway in PAECs was chronicled in patients with PAH and in experimental pulmonary hypertension rodent models subjected to monocrotaline (MCT), chronic hypoxia, and SU5416/hypoxia (Chandra et al., 2011; Kim et al., 2013). In addition, apelin knockout mice developed more severe PAH compared with wild-type mice, and displayed significant loss of pulmonary microvasculature when subjected to chronic hypoxia (Chandra et al., 2011). More importantly, other studies have reported significantly reductions of apelin levels in the serum and pulmonary endothelium of patients with $\mathrm{PAH}$, as well as in the lungs of rats with MCT-induced pulmonary hypertension (Alastalo et al., 2011; Chandra et al., 2011; Falcão-Pires et al., 2009; Goetze et al., 2006; Kim et al., 2013). Furthermore, PAH in both the MCT model and endothelial-specific PPARy knockout mice, in which apelin is reduced, is reversed by exogenous apelin peptide administration (Alastalo et al., 2011; Chandra et al., 2011; Falcão-Pires et al., 2009; Kim et al., 2013). The findings of hyperproliferative and antiapoptotic activity of PAH PAECs (Kim et al., 2013; Masri et al., 2007; Tuder et al., 1994), in conjunction with other studies showing decreased apelin expression in PAH (Alastalo et al., 2011; Chandra et al., 2011; Goetze et al., 2006; Kim et al., 2013) supports the view that disruption of apelin-APJ signaling in PAH contributes to the aberrant activation of a downstream secondary signaling cascade that induces the abnormal proliferation of pulmonary vascular cells, leading to progressive vascular remodeling.

Downstream signal transduction of apelin-APJ signaling in $\mathrm{PAH}$

Given that the apelin-APJ signaling is disrupted in PAH and 
A

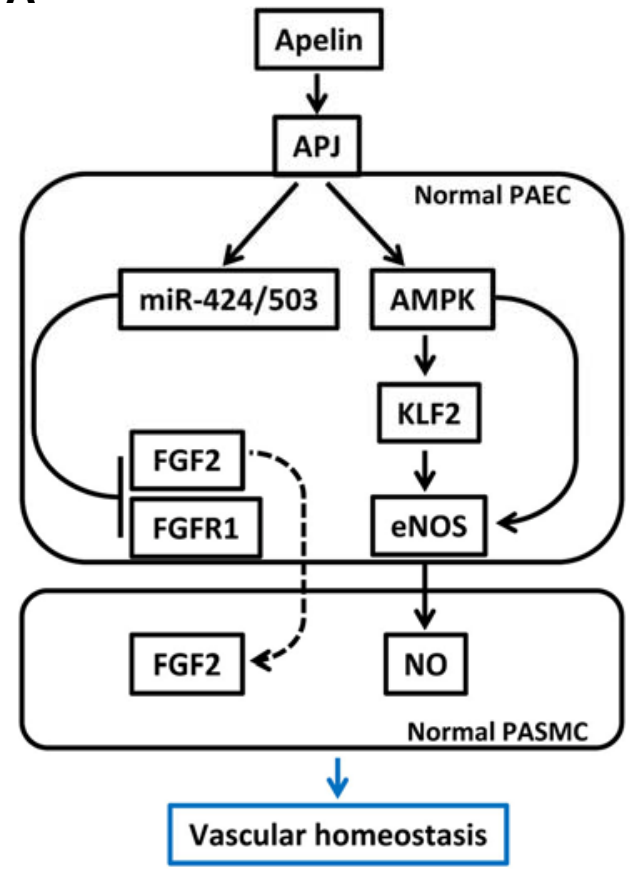

B

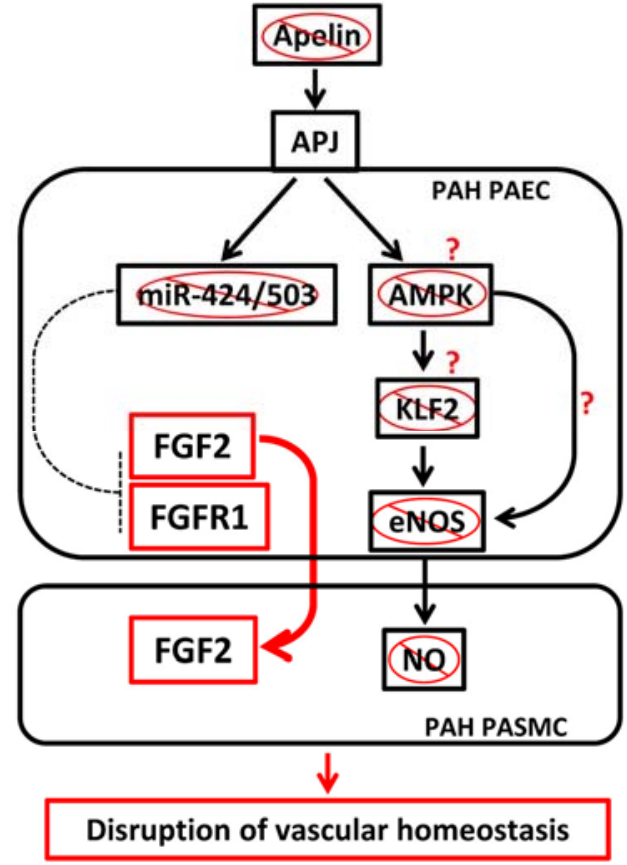

Fig. 2. Putative model of the mechanism of vascular homeostasis mediated by apelin-APJ signaling. (A) Basal apelin-APJ signaling maintains expression of miR424/503, KLF2, and eNOS, as well as activity of AMPK. miR-424/503 in turn inhibits FGF2/FGFR1 expression, and eNOS induces the production of NO. (B) In PAH, there is decreased apelin, $\mathrm{miR}$ 424/503 and eNOS expression, leading to abnormal activation of FGF signaling pathway and reducing NO level that target both the PAECs and PASMCs. represents a novel, potentially fruitful therapeutic target, pursuing various downstream signaling mechanisms that mediate the vascular protective effects of apelin-APJ signaling could yield important mechanistic data. The apelin-APJ pathway targets critical mediators of endothelial homeostasis, including KLF2, (Chandra et al., 2011; McLean et al., 2012) and endothelial nitric oxide synthase (eNOS) (Chandra et al., 2011). A potential role of eNOS in PAH is supported by the finding of decreased expression of eNOS in the lungs of PAH patients (Chandra et al., 2011; Giaid and Saleh, 1995). Apelin deficient mice are more susceptible to hypoxia-induced $\mathrm{PAH}$, at least in part secondary to decreased eNOS expression in the lung of apelin-null mice as well as in the PAECs with apelin knockdown, leading to decreased NO production (Chandra et al., 2011). Although KLF2 regulation of eNOS expression has been well established (Lin et al., 2005; Sen-Banerjee et al., 2005), AMPK and KLF2 appear to be critical intermediaries for apelin/APJ regulation of eNOS expression in vitro knockdown studies.

NO regulated by the apelin-APJ-AMPK-KLF2-eNOS axis plays an important role in normal pulmonary vascular homeostasis and is a mediator of pulmonary vascular remodeling. It is a potent pulmonary vasodilator produced locally in the lungs and mediates smooth muscle relaxation and proliferation. eNOS catalyzes the conversion of L-arginine to citrulline, producing $\mathrm{NO}$ in a number of tissues, including the lungs. NO diffuses into PASMCs, leading to production of guanosine $3^{\prime}, 5^{\prime}$-cyclic monophosphate (cGMP) (Moncada and Higgs, 2006) via activation of guanylate cyclase. In turn, increased cGMP leads to hyperpolarization and PASMC relaxation. Patients with $\mathrm{PAH}$ have low levels of NO in their exhaled breath (Kaneko et al., 1998). Although NO administration is effective therapy for $\mathrm{PAH}$, technical limitations of gas delivery have limited widespread use (Chan-nick et al., 1996; Rubin, 1997). Rather, multiple therapies may use NO as a mediator of their efficacy. For example, infusion of epoprostenol, a prostacyclin analogue, increases the
NO levels in the exhaled breath of patients (Ozkan et al., 2001) (Fig. 2).

MiRNAs are a class of small, noncoding RNAs that have critical post-transcriptional regulatory roles targeting mRNA with their main function being down-regulation of gene expression (Kim, 2005). MiRNAs are emerging as key, powerful transacting factors that regulate gene expression and fundamental cellular processes (Kim and Kim, 2012), and which may play an important role in the pathogenesis of $\mathrm{PAH}$, including miR-17, miR-20a, and miR-21 (Brock et al., 2012; Parikh et al., 2012; Pullamsetti et al., 2012). In addition, a recent study identified two key endothelial miRNAs, miR-424 and miR-503, which are regulated by apelin-APJ signaling and which target two molecules of the FGF signaling pathway (FGF2 and fibroblast growth factor receptor 1, FGFR1), associated with the cellular hyperproliferation and vascular remodeling found in $\mathrm{PAH}$. These miRNAs are highly expressed in normal PAECs, but are also markedly decreased in PAECs isolated from patients with clinically diagnosed PAH (Kim et al., 2013). FGF2 expression is increased in PAECs from PAH patients (Izikki et al., 2009). Kim et al. (2013) also demonstrated that restoration of miR-424/503 expression in the experimental PAH models by intranasal lentiviral delivery can significantly ameliorate the severity of the disease through reversal of a progressive vascular remodeling by inhibition of abnormal proliferation of PAECs and PASMCs, suggesting that they may serve as potential therapeutic agents in clinical PAH (Fig. 2). The existing approved therapies primarily target achieving vasodilatation, whereas emerging data suggest that targeting abnormal proliferation of the vascular cells may be a viable therapeutic venue.

In the context of the endothelium, a number of miRNAs have emerged as critical regulators of cellular development and function (Urbich et al., 2004). However, the elucidation of their role in the PAECs and $\mathrm{PAH}$ is in its infancy. Further characterization of their involvement in $\mathrm{PAH}$ will provide new insight and poten- 
tially novel therapeutic targets for this deadly disease through reversal of the hyperproliferative, antiapoptotic phenotype of the PAECs associated with PAH. Given that apelin-APJ signaling could regulate various downstream molecules, such as KLF2, eNOS and miR-424/503, that are able to modulate the vascular remodeling in $\mathrm{PAH}$, apelin-APJ signaling is a very attractive therapeutic target for PAH therapy (Fig. 1).

\section{CONCLUSIONS}

In summary, $\mathrm{PAH}$ is characterized by vascular remodeling resulting from hyperproliferation of PAECs and PASMC in the pulmonary vasculature. Disruption of apelin-APJ signaling appears to play a major pathogenic role in these events. This view is also supported by restoration effects of $\mathrm{PAH}$ animal model caused by rescue of disrupted apelin signaling. Emerging evidence indicates that apelin-APJ signaling targets various key genes, such as eNOS, KLF2, miR-424, and miR-503, involved in the pathogenesis of $\mathrm{PAH}$. Although apelin-APJ signalingrelated targets in $\mathrm{PAH}$ need further elucidation, apelin may affect both reversal pulmonary vascular remodeling through the antiproliferatory effect of PAECs and PASMCs and its properties as a vasodilator, implicating it as a potentially useful therapeutic target in the treatment of $\mathrm{PAH}$. Ongoing studies will yield substantial increase in our understanding of the molecular actions of various targets of apelin-APJ signaling in $\mathrm{PAH}$.

\section{ACKNOWLEDGMENTS}

I thank Dr. Hyung J. Chun, assistant professor of the Yale University School of Medicine, for critical reading of the manuscript and thoughtful discussions. This Research was supported by the Sookmyung Women's University Research Grants of 11303-0061

\section{REFERENCES}

Alastalo, T.P., Li, M., Perez Vde, J., Pham, D., Sawada, H., Wang, J.K., Koskenvuo, M., Wang, L., Freeman, B.A., Chang, H.Y., et al. (2011). Disruption of PPAR $\gamma / \beta$-catenin-mediated regulation of apelin impairs BMP-induced mouse and human pulmonary arterial EC survival. J. Clin. Invest. 121, 3735-3746.

Ashley, E.A., Powers, J., Chen, M., Kundu, R., Finsterbach, T., Caffarelli, A., Deng, A., Eichhorn, J., Mahajan, R., Agrawal, R., et al. (2005). The endogenous peptide apelin potently improves cardiac contractility and reduces cardiac loading in vivo. Cardiovasc. Res. 65, 73-82.

Barst, R.J. (2005). PDGF signaling in pulmonary arterial hypertension. J. Clin. Invest. 115, 2691-2694.

Benisty, J.I., McLaughlin, V.V., Landzberg, M.J., Rich, J.D., Newburger, J.W., Rich, S., and Folkman, J. (2004). Elevated basic fibroblast growth factor levels in patients with pulmonary arterial hypertension. Chest 126, 1255-1261.

Brock, M., Samillan, V.J., Trenkmann, M., Schwarzwald, C., Ulrich, S., Gay, R.E., Gassmann, M., Ostergaard, L., Gay, S., Speich, R., et al. (2012). AntagomiR directed against miR-20a restores functional BMPR2 signalling and prevents vascular remodelling in hypoxia-induced pulmonary hypertension. Eur. Heart J. doi: 10.1093/eurhearti/ehs060.

Budhiraja, R., Tuder, R.M., and Hassoun, P.M. (2004). Endothelial dysfunction in pulmonary hypertension. Circulation 109, 159-165

Chandra, S.M., Razavi, H., Kim, J., Agrawal, R., Kundu, R.K., de Jesus Perez, V., Zamanian, R.T., Quertermous, T., and Chun, H.J. (2011). Disruption of the apelin-APJ system worsens hypoxia-induced pulmonary hypertension. Arterioscler. Thromb. Vasc. Biol. 31, 814-820.

Chen, M.M., Ashley, E.A., Deng, D.X., Tsalenko, A., Deng, A., Tabibiazar, R., Ben-Dor, A., Fenster, B., Yang, E., King, J.Y., et al. (2003). Novel role for the potent endogenous inotrope apelin in human cardiac dysfunction. Circulation 108, 1432-1439.
Cheng, X., Cheng, X.S., and Pang, C.C. (2003). Venous dilator effect of apelin, an endogenous peptide ligand for the orphan APJ receptor, in conscious rats. Eur. J. Pharmacol. 470, 171-175.

Chun, H.J., Ali, Z.A., Kojima, Y., Kundu, R.K., Sheikh, A.Y., Agrawal, R., Zheng, L., Leeper, N.J., Pearl, N.E., Patterson, A.J., et al. (2008). Apelin signaling antagonizes Ang II effects in mouse models of atherosclerosis. J. Clin. Invest. 118, 3343-3354.

De Mota, N., Reaux-Le Goazigo, A., El Messari, S., Chartrel, N. Roesch, D., Dujardin, C., Kordon, C., Vaudry, H., Moos, F., and Llorens-Cortes, C. (2004). Apelin, a potent diuretic neuropeptide counteracting vasopressin actions through inhibition of vasopressin neuron activity and vasopressin release. Proc. Natl. Acad. Sci. USA 101, 10464-10469.

Deng, Z., Morse, J.H., Slager, S.L., Cuervo, N., Moore, K.J., Venetos, G., Kalachikov, S., Cayanis, E., Fischer, S.G., Barst, R.J., et al. (2000). Familial primary pulmonary hypertension (gene PPH1) is caused by mutations in the bone morphogenetic protein receptor-II gene. Am. J. Hum. Genet. 67, 737-744.

Falcão-Pires, I., Gonçalves, N., Henriques-Coelho, T., MoreiraGonçalves, D., Roncon-Albuquerque, R. Jr., and Leite-Moreira A.F. (2009). Apelin decreases myocardial injury and improves right ventricular function in monocrotaline-induced pulmonary hypertension. Am. J. Physiol. Heart Circ. Physiol. 296, H2007H2014.

Ghofrani, H.A., Morrell, N.W., Hoeper, M.M., Olschewski, H., Peacock, A.J., Barst, R.J., Shapiro, S., Golpon, H., Toshner, M., Grimminger, F., et al. (2010). Imatinib in pulmonary arterial hypertension patients with inadequate response to established therapy. Am. J. Respir. Crit. Care Med. 182, 1171-1177.

Giaid, A., and Saleh, D. (1995). Reduced expression of endothelial nitric oxide synthase in the lungs of patients with pulmonary hypertension. N. Engl. J. Med. 333, 214-221.

Goetze, J.P., Rehfeld, J.F., Carlsen, J., Videbaek, R., Andersen, C. B., Boesgaard, S., and Friis-Hansen, L. (2006). Apelin: a new plasma marker of cardiopulmonary disease. Regul. Pept. 133, 134-138.

Hamid, R., Cogan, J.D., Hedges, L.K., Austin, E., Phillips, J.A. 3rd, Newman, J.H., and Loyd, J.E. (2009). Penetrance of pulmonary arterial hypertension is modulated by the expression of normal BMPR2 allele. Hum. Mutat. 30, 649-654.

Hanahan, D., and Weinberg, R.A. (2000). The hallmarks of cancer. Cell 100, 57-70.

Hansmann, G., Wagner, R.A., Schellong, S., Perez, V.A., Urashima T., Wang, L., Sheikh, A.Y., Suen, R.S., Stewart, D.J., and Rabinovitch, M. (2007). Pulmonary arterial hypertension is linked to insulin resistance and reversed by peroxisome proliferatoractivated receptor-gamma activation. Circulation 115, 1275-1284.

Hosoya, M., Kawamata, Y., Fukusumi, S., Fujii, R., Habata, Y. Hinuma, S., Kitada, C., Honda, S., Kurokawa, T., Onda, H., et al. (2000). Molecular and functional characteristics of APJ. Tissue distribution of mRNA and interaction with the endogenous ligand apelin. J. Biol. Chem. 275, 21061-21067.

Humbert, M., Sitbon, O., Chaouat, A., Bertocchi, M., Habib, G., Gressin, V., Yaïci, A., Weitzenblum, E., Cordier, J.F., Chabot, F., et al. (2010). Survival in patients with idiopathic, familial, and anorexigen-associated pulmonary arterial hypertension in the modern management era. Circulation 122, 156-163.

Ishida, J., Hashimoto, T., Hashimoto, Y., Nishiwaki, S., Iguchi, T., Harada, S., Sugaya, T., Matsuzaki, H., Yamamoto, R., Shiota, N., et al. (2004). Regulatory roles for APJ, a seven-transmembrane receptor related to angiotensin-type 1 receptor in blood pressure in vivo. J. Biol. Chem. 279, 26274-26279.

Iwanaga, Y., Kihara, Y., Takenaka, H., and Kita, T. (2006). Downregulation of cardiac apelin system in hypertrophied and failing hearts: Possible role of angiotensin II-angiotensin type 1 receptor system. J. Mol. Cell Cardiol. 41, 798-806.

Izikki, M., Guignabert, C., Fadel, E., Humbert, M., Tu, L., Zadigue, P., Dartevelle, P., Simonneau, G., Adnot, S., Maitre, B., et al. (2009). Endothelial-derived FGF2 contributes to the progression of pulmonary hypertension in humans and rodents. J. Clin. Invest. 119, 512-523.

Kaneko, F.T., Arroliga, A.C., Dweik, R.A., Comhair, S.A., Laskowski, D., Oppedisano, R., Thomassen, M.J., and Erzurum, S.C. (1998) Biochemical reaction products of nitric oxide as quantitative markers of primary pulmonary hypertension. Am. J. Respir. Crit. Care Med. 158, 917-923.

Kang, Y., Kim, J., Anderson, J.P., Wu, J., Gleim, S.R., Kundu, R.K., 
McLean, D.L., Kim, J.D., Park, H., Jin, S.W., et al. (2013). Apelin-APJ signaling is a critical regulator of endothelial MEF2 activation in cardiovascular development. Circ. Res. 113, 22-31.

Kawamata, Y., Habata, Y., Fukusumi, S., Hosoya, M., Fujii, R., Hinuma, S., Nishizawa, N., Kitada, C., Onda, H., Nishimura, O., et al. (2001). Molecular properties of apelin: tissue distribution and receptor binding. Biochim. Biophys. Acta 1538, 162-171.

Kim, V.N. (2005). Small RNAs: classification, biogenesis, and function. Mol. Cells 19, 1-15.

Kim, Y., and Kim, V.N. (2012). MicroRNA factory: RISC assembly from precursor microRNAs. Mol. Cell 46, 384-386.

Kim, J., Kang, Y., Kojima, Y., Lighthouse, J.K., Hu, X., Aldred, M.A., McLean, D.L., Park, H., Comhair, S.A., Greif, D.M., et al. (2013). An endothelial apelin-FGF link mediated by miR-424 and miR503 is disrupted in pulmonary arterial hypertension. Nat. Med. 19, 74-82.

Lee, D.K., Cheng, R., Nguyen, T., Fan, T., Kariyawasam, A.P., Liu, Y., Osmond, D.H., George, S.R., and O'Dowd, B.F. (2000). Characterization of apelin, the ligand for the APJ receptor. J. Neurochem. 74, 34-41.

Lin, Z., Kumar, A., SenBanerjee, S., Staniszewski, K., Parmar, K., Vaughan, D.E., Gimbrone, M.A. Jr., Balasubramanian, V., GarcíaCardeña, G., and Jain, M.K. (2005). Kruppel-like factor 2 (KLF2) regulates endothelial thrombotic function. Circ. Res. 96, e48-e57.

Machado, R.D., Eickelberg, O., Elliott, C.G., Geraci, M.W., Hanaoka, M., Loyd, J.E., Newman, J.H., Phillips, J.A. 3rd., Soubrier, F., Trembath, R.C., et al. (2009). Genetics and genomics of pulmonary arterial hypertension. J. Am. Coll. Cardiol. 54, S32-S42.

Masri, F.A., Xu, W., Comhair, S.A., Asosingh, K., Koo, M., Vasanji, A., Drazba, J., Anand-Apte, B., and Erzurum, S.C. (2007). Hyperproliferative apoptosis-resistant endothelial cells in idiopathic pulmonary arterial hypertension. Am. J. Physiol. Lung Cell Mol. Physiol. 293, L548-L554

McLaughlin, V.V., and McGoon, M.D. (2006). Pulmonary arterial hypertension. Circulation 114, 1417-1431.

McLean, D.L., Kim, J., Kang, Y., Shi, H., Atkins, G.B., Jain, M.K., and Chun, H.J. (2012). Apelin/APJ signaling is a critical regulator of statin effects in vascular endothelial cells. Arterioscler. Thromb. Vasc. Biol. 32, 2640-2643.

Medhurst, A.D., Jennings, C.A., Robbins, M.J., Davis, R.P., Ellis, C., Winborn, K.Y., Lawrie, K.W., Hervieu, G., Riley, G., Bolaky, J.E., et al. (2003). Pharmacological and immunohistochemical characterization of the APJ receptor and its endogenous ligand apelin. J. Neurochem. 84, 1162-1172.

Moncada, S., and Higgs, E.A. (2006). The discovery of nitric oxide and its role in vascular biology. Br. J. Pharmacol. 147 Suppl 1, S193-S201.

O'Carroll, A.M., Lolait, S.J., and Howell, G.M. (2006). Transcriptional regulation of the rat apelin receptor gene: promoter cloning and identification of an Sp1 site necessary for promoter activity. J. Mol. Endocrinol. 36, 221-235.

O'Dowd, B.F., Heiber, M., Chan, A., Heng, H.H., Tsui, L.C., Kennedy, J.L., Shi, X., Petronis, A., George, S.R., and Nguyen, T. (1993). A human gene that shows identity with the gene encoding the angiotensin receptor is located on chromosome 11. Gene 136, 355-360.

Ozkan, M., Dweik, R.A., Laskowski, D., Arroliga, A.C., and Erzurum, S.C. (2001). High levels of nitric oxide in individuals with pulmonary hypertension receiving epoprostenol therapy. Lung 179, 233-243.

Parikh, V.N., Jin, R.C., Rabello, S., Gulbahce, N., White, K., Hale, A., Cottrill, K.A., Shaik, R.S., Waxman, A.B., Zhang, Y.Y., et al. (2012). MicroRNA-21 integrates pathogenic signaling to control pulmonary hypertension: results of a network bioinformatics approach. Circulation 125, 1520-1532.

Pullamsetti, S.S., Doebele, C., Fischer, A., Savai, R., Kojonazarov,
B., Dahal, B.K., Ghofrani, H.A., Weissmann, N., Grimminger, F. Bonauer, A., et al. (2012). Inhibition of microRNA-17 improves lung and heart function in experimental pulmonary hypertension. Am. J. Respir. Crit. Care Med. 185, 409-419.

Rabinovitch, M. (2007). Pathobiology of pulmonary hypertension. Annu. Rev. Pathol. 2, 369-399.

Rai, P.R., Cool, C.D., King, J.A., Stevens, T., Burns, N., Winn, R.A., Kasper, M., and Voelkel, N.F. (2008). The cancer paradigm of severe pulmonary arterial hypertension. Am. J. Respir. Crit. Care Med. 178, 558-564.

Raja, S.G. (2010). Endothelin receptor antagonists for pulmonary arterial hypertension: an overview. Cardiovasc. Ther. 28, e65-e71.

Roberts, K.E., McElroy, J.J., Wong, W.P., Yen, E., Widlitz, A., Barst, R.J., Knowles, J.A., and Morse, J.H. (2004). BMPR2 mutations in pulmonary arterial hypertension with congenital heart disease. Eur. Respir. J. 24, 371-374.

Rubin, L.J. (1997). Primary pulmonary hypertension. N. Engl. J. Med. 336, 111-117.

Sakao, S., Tatsumi, K., and Voelkel, N.F. (2010). Reversible or irreversible remodeling in pulmonary arterial hypertension. Am. J. Respir. Cell Mol. Biol. 43, 629-634.

Sen-Banerjee, S., Mir, S., Lin, Z., Hamik, A., Atkins, G.B., Das, H., Banerjee, P., Kumar, A., and Jain, M.K. (2005). Kruppel-like factor 2 as a novel mediator of statin effects in endothelial cells. Circulation 112, 720-726.

Sheikh, A.Y., Chun, H.J., Glassford, A.J., Kundu, R.K., Kutschka, I. Ardigo, D., Hendry, S.L., Wagner, R.A., Chen, M.M., Ali, Z.A., et al. (2008). In vivo genetic profiling and cellular localization of apelin reveals a hypoxia-sensitive, endothelial-centered pathway activated in ischemic heart failure. Am. J. Physiol. Heart Circ. Physiol. 294, H88-H98.

Simonneau, G., Robbins, I.M., Beghetti, M., Channick, R.N., Delcroix, M., Denton, C.P., Elliott, C.G., Gaine, S.P., Gladwin, M.T., Jing, Z.C., et al. (2009). Updated clinical classification of pulmonary hypertension. J. Am. Coll. Cardiol. 54, S43-S54.

Szokodi, I., Tavi, P., Földes, G., Voutilainen-Myllylä, S., Ilves, M., Tokola, H., Pikkarainen, S., Piuhola, J., Rysä, J., Tóth, M., et al. (2002). Apelin, the novel endogenous ligand of the orphan receptor APJ, regulates cardiac contractility. Circ. Res. 91, 434-440.

Tatemoto, K., Hosoya, M., Habata, Y., Fujii, R., Kakegawa, T., Zou, M.X., Kawamata, Y., Fukusumi, S., Hinuma, S., Kitada, C., et al. (1998). Isolation and characterization of a novel endogenous peptide ligand for the human APJ receptor. Biochem. Biophys. Res. Commun. 251, 471-476.

Tatemoto, K., Takayama, K., Zou, M.X., Kumaki, I., Zhang, W., Kumano, K., and Fujimiya, M. (2001). The novel peptide apelin lowers blood pressure via a nitric oxide-dependent mechanism. Regul. Pept. 99, 87-92.

Tuder, R.M., Groves, B., Badesch, D.B., and Voelkel, N.F. (1994). Exuberant endothelial cell growth and elements of inflammation are present in plexiform lesions of pulmonary hypertension. Am. J. Pathol. 144, 275-285.

Tuder, R.M., Cool, C.D., Yeager, M., Taraseviciene-Stewart, L., Bull, T.M., and Voelkel, N.F. (2001). The pathobiology of pulmonary hypertension. Endothelium Clin. Chest Med. 22, 405-418.

Urbich, C., Kuehbacher, A., and Dimmeler, S. (2008). Role of microRNAs in vascular diseases, inflammation, and angiogenesis. Cardiovasc. Res. 79, 581-588.

Zaidi, S.H., You, X.M., Ciura, S., Husain, M., and Rabinovitch, M. (2002). Overexpression of the serine elastase inhibitor elafin protects transgenic mice from hypoxic pulmonary hypertension. Circulation 105, 516-521.

Zamanian, R.T., Hansmann, G., Snook, S., Lilienfeld, D., Rappaport, K.M., Reaven, G.M., Rabinovitch, M., and Doyle, R.L. (2009) Insulin resistance in pulmonary arterial hypertension. Eur. Respir. J. 33, 318-324. 